Pacific Journal of Mathematic

roo sulfur 


\title{
SUBCONTINUITY FOR MULTIFUNCTIONS
}

\author{
R. E. SMITHSON
}

The concept of subcontinuity is extended to multifunctions. This notion is then used to obtain a number of results on multifunctions with closed graphs and to develop criteria under which a multifunction is upper semicontinuous.

1. Introduction. In [1] R. V. Fuller introduced the concept of subcontinuous function and used it to obtain conditions implying continuity as well as some comparisons to compactness preserving functions and some results on functions with closed graphs. In [1] Fuller stated that he thought that his results would hold for multifunctions. The purpose of this paper is to show that such is the case. Indeed the definition of subcontinuity can be extended and conditions implying upper semi-continuity for multifunctions are derived, including a generalization of a result of Muenzenberger and Smithson [4].

By a multifunction we mean a correspondence $F: X \rightarrow Y$ on a set $X$ into a set $Y$ such that $F(x)$ is a nonempty subset of $Y$ for each $x \in X$. If $F$ is a multifunction, then the graph of $F$ is the subset $\{(x, y): x \in X, y \in F(x)\}$ of $X \times Y$. We denote the graph of $F$ by $G(F)$. Then a multifunction $F$ has a closed graph if $G(F)$ is a closed subset of $X \times Y$. Whereas $F$ is a closed multifunction if $F(A)$ is closed in $Y$ for all closed sets $A \subset X$. Further, $F$ is upper semicontinuous (u.s.c.) if and only if for each closed set $B \subset Y, F^{-1}(B)=$ $\{x: F(x) \cap B \neq \varnothing\}$ is closed. This is equivalent to saying that $F^{-1}$ (where $F^{-1}(y)=\{x: y \in F(x)\}$ ) is a closed multifunction on $F(X)=$ $\bigcup\{F(x): x \in X\}$.

If $A \subset X$, then $A^{-}$denotes the closure of $A$. Other notation used is that of Kelley [3].

2. Subcontinuous multifunctions. Using Fuller's definition as a model we obtain the following definition.

Definition. A multifunction $F: X \rightarrow Y$ is subcontinuous if and only if whenever $\left\{x_{\alpha}, \alpha \in D\right\}$ is a convergent net in $X$ and $\left\{y_{\alpha}, \alpha \in D\right\}$ is a net in $F(X)$ with $y_{\alpha} \in F\left(x_{\alpha}\right)$ then $\left\{y_{\alpha}, \alpha \in D\right\}$ has a convergent subnet.

Next we say that $F$ is inversely subcontinuous in case $F^{-1}$ is subcontinuous. Note that since $F$ and $F^{-1}$ are both multifunctions we did not need a separate definition in terms of nets for inverse subcontinuity. Also, if $F$ is single valued then the present definitions 
are equivalent to Fuller's definitions. Finally, since the concepts of subcontinuity and inverse subcontinuity are dual, a result about one also has a dual result for the other. In most cases we shall give only the result for subcontinuous functions.

Immediate consequences of the definition of subcontinuous multifunction $F$ are: If $K \subset X$ is compact and if $F(K)$ is closed, then $F(K)$ is compact. That is, in this case $F$ preserves compact sets. Consequently a point closed (i.e. $F(x)$ closed for each $x \in X$ ) subcontinuous multifunction is point compact (i.e. $F(x)$ is compact for each $x \in X)$.

THEOREM 2.1. If $F: X \rightarrow Y$ is a subcontinuous multifunction on the space $X$ into the completely regular space $Y$ and if $K \subset X$ is compact, then $F(K)^{-}$is compact.

Proof. Suppose $K \subset X$ is compact and let $\left\{y_{\alpha}, \alpha \in D\right)$ be a net in $F(K)$. Let $\mathscr{B}$ be a base for the uniformity on $Y$ and partial order $\mathscr{B}$ by inclusion downward. Then give $D \times \mathscr{B}$ the product partial order. Then for each $a=(\alpha, B) \in D \times \mathscr{B}$ pick an element $z_{a}$ in $B\left[y_{\alpha}\right] \cap F(K)$. Next let $x_{a}$ be an element of $K$ such that $z_{a} \in F\left(x_{a}\right)$. Now since $K$ is compact some subnet $\left\{x_{a_{n}}\right\}$ of $\left\{x_{a}, a \in D \times \mathscr{B}\right\}$ converges and consequently some subnet of $\left\{z_{a_{n}}\right\}$ say $\left\{z_{a_{m}}\right\}$ converges, since $F$ is subcontinuous, to $z_{0}$. Now $a_{m}=\left(\alpha_{m}, B_{m}\right)$ and $z_{a_{m}} \in B_{m}\left[y_{\alpha_{m}}\right]$. Next let $U$ be a neighborhood of $z_{0}$ and let $B$ be a symmetric member of $\mathscr{B}$ such that $B \circ B\left[z_{0}\right] \subset U$. There is an $a_{m^{\prime}}$ such that $a_{m^{\prime}}<a_{m}$ (i.e. $\alpha_{m^{\prime}}<\alpha_{m}$ and $\left.B_{m} \subset B_{m^{\prime}}\right)$ implies that $z_{a_{m}} \in B\left[z_{0}\right]$ and since $z_{m}$ is a subnet of $z_{a}, B_{m} \subset B$. Now $\left(z_{0}, z_{a_{m}}\right) \in B$ and $z_{a_{m}} \in B_{m}\left[y_{\alpha_{m}}\right]$ so $\left(y_{\alpha_{m}}, z_{a_{m}}\right) \in B_{m} \subset B$ and since $B$ is symmetric $\left(z_{0}, y_{\alpha_{m}}\right) \in B \circ B$. Thus $y_{\alpha_{m}} \in B \circ B\left[z_{0}\right] \subset U$. Hence, a subnet of $\left\{y_{\alpha}, \alpha \in D\right\}$ converges.

Next we characterize compact preserving multifunctions in terms of subcontinuity.

Theorem 2.2. Let $F: X \rightarrow Y$ be a multifunction. Then $F$ is compact preserving if and only if $F \mid K \rightarrow F(K)$ is subcontinuous for each compact set $K \subset X$.

Proof. Suppose $F$ is compact preserving and let $K \subset X$ be compact. Then $F(K)$ is compact and consequently $F \mid K \rightarrow F(K)$ is subcontinuous.

Next let $K \subset X$ be compact and suppose $F \mid K \rightarrow F(K)$ is subcontinuous. But then each set in $F(K)$ must have a convergent subnet and thus $F(K)$ is compact. Hence, $F$ is compact preserving.

3. Closed graphs and upper semicontinuity. A number of 
examples exist which show that a function with a closed graph need not be continuous. However, it is easy to show that a function with a closed graph into a compact space is continuous (see remark below). By using subcontinuity we can get a significant generalization of this.

THEOREM 3.1. Let $F: X \rightarrow Y$ be a subcontinuous multifunction which has a closed graph. Then $F$ is upper semicontinuous.

Proof. Let $A \subset Y$ be closed and let $x_{0} \in F^{-1}(A)^{-}$. Further, let $\left\{x_{\alpha}, \alpha \in D\right\}$ be a net in $F^{-1}(A)$ which converges to $x_{0}$. Let $\left\{y_{\alpha}, \alpha \in D\right\}$ be net in $A$ such that $y_{\alpha} \in F\left(x_{\alpha}\right)$ for each $\alpha$. Then, since $F$ is subcontinuous, there is a convergent subnet $y_{\alpha_{n}} \rightarrow y_{0} \in A$. If $y_{0} \notin F\left(x_{0}\right)$, then $\left(x_{0}, y_{0}\right) \notin G(F)$ and since $G(F)$ is closed, there exist open sets $U \subset X, V \subset Y$, such that $\left(x_{0}, y_{0}\right) \in U \times V \subset X \times Y \backslash G(F)$. But $x_{\alpha} \rightarrow x_{0}$ and $y_{\alpha_{n}} \rightarrow y_{0}$. Thus there is an $\alpha_{n}$ such that $x_{\alpha_{n}} \in U$ and $y_{\alpha_{n}} \in V$ which is a contradiction. Thus $y_{0} \in F\left(x_{0}\right)$ and $x_{0} \in F^{-1}(A)$. Hence, $F$ is u.s.c.

We can deduce another result from the proof of Theorem 3.1. This result strengthens known results on upper semicontinuous point closed multifunctions.

Proposition 3.2. Let $F: X \rightarrow Y$ be a subcontinuous multifunction with a closed graph. Let $x_{\alpha} \rightarrow x_{0}$ and let $\left\{y_{\alpha}\right\}$ be a net such that $y_{\alpha} \in F\left(x_{\alpha}\right)$ for each $\alpha$. If $y_{\alpha} \rightarrow y_{0}$, then $y_{0} \in F\left(x_{0}\right)$.

If we assume that $Y$ is regular in Theorem 3.1, then the converse is also true.

THEOREM 3.3. If $F: X \rightarrow Y$ is a point closed upper semicontinuous multifunction into a regular space, then $F$ has a closed graph.

Proof. Suppose $(x, y) \notin G(F)$. Then $y \notin F(x)$. Thus there are disjoint open sets $U, V \subset Y$ such that $F(x) \subset U$ and $y \in V$. Further, there is an open set $W \subset X$ with $x \in W$ such that $F(W) \subset U$ ( $W$ exists because $F$ is u.s.c.). Thus $(x, y) \in W \times V$ and $W \times V \cap G(F)=\varnothing$. Hence, $G(f)$ is closed.

REMARK. In [1] Fuller had a result for single valued functions which is analogous to Theorem 3.3. In this result Fuller assumed that $Y$ was Hausdorff. But in the case of multifunctions we need to assume that $Y$ is regular.

As we have seen a multifunction with a closed graph is in a sense almost u.s.c. In this regard there is an exercise in Kelley [3] which asserts that for such a function $F(K)$ is closed and $F^{-1}\left(K^{\prime}\right)$ is 
closed whenever $K, K^{\prime}$ are compact (a proof of this appears in [4]). Next we develop some conditions under which a multifunction has a closed graph. For this we need the following definition.

Definition. A multifunction $F: X \rightarrow Y$ is called locally closed if and only if for each $p \in X$ and for each neighborhood $U$ of $p$ there is a neighborhood $V$ of $p$ such that $V \subset U$ and $F(V)$ is closed.

A closed function or multifunction on a regular space is locally closed. But a locally closed function need not be closed.

TheOREM 3.4. If $F: X \rightarrow Y$ is locally closed and if $F^{-1}(y)$ is closed for each $y \in Y$, then $F$ has a closed graph.

Proof. If $y \notin F(x)$, then $x \notin F^{-1}(y)$ and so there is a neighborhood $U$ of $x$ such that $U \cap F^{-1}(y)=\phi$ and $F(U)$ is closed. Then there is an open set $V$ with $y \in V$ such that $F(U) \cap V=\varnothing$. Then $U \times V$ is a neighborhood of $(x, y)$ which misses $G(F)$. Hence, $F$ has a closed graph.

Corollary 3.5. If $F: X \rightarrow Y$ is a closed multifunction on a regular space $X$ and if $F^{-1}(y)$ is closed for each $y \in Y$, then $F$ has a closed graph.

REMARK. Corollary 3.5 is a restatement of Theorem 3.3.

Since a closed multifunction on a regular space is locally closed, and since a multifunction with compact range is subcontinuous; Theorems 3.1 and 3.4 given the following result from [5] which was a generalization of a result of Halfar [2].

CoROllary 3.6. If $F: X \rightarrow Y$ is a closed multifunction on a regular space $X$ into a compact space $Y$ for which $F^{-1}(y)$ is closed for all $y \in Y$, then $F$ is upper semicontinuous.

Another immediate consequence of the definition of locally closed is that a locally closed multifunction with closed point inverses is point closed.

By putting together some of the above we can prove.

THeOREM 3.7. If $X$ is a regular, locally compact space, and if $F$ is a multifunction on $F$, then the following are equivalent.

(i) $F$ has a closed graph

(ii) If $K \subset X$ is compact, then $F(K)$ and $F^{-1}(y)$ are closed for each $y \in Y$. 
(iii) $F$ is locally closed and $F^{-1}(y)$ is closed for each $y \in Y$.

Proof. That (iii) implies (i) is Theorem 3.4 and (i) implies (ii) was proved in [4]. Finally, since $X$ is regular and locally compact, $X$ has a compact bases for each point and hence, (ii) implies (iii).

4. Compact preserving multifunctions and $k$-spaces. In [1] Fuller defines what he calls a $k_{3}$-space. This definition is only slightly different from the definition of a $k$-space as given by Kelley [3]. In fact when the space is Hausdorff they are identical. We shall use the definition given by Fuller.

Definition. A topological space $X$ is a $k_{3}$-space provided that a set is open in $X$ if and only if its intersection with each compact subset of $X$ is relatively open.

Our next theorem gives an extension of the main result in [4].

THEOREM 4.1. Let $F: X \rightarrow Y$ be a multifunction on a $k_{3}$-space. If $F$ is compact preserving and if $F$ has a closed graph, then $F$ is upper semicontinuous.

Proof. Let $A \subset Y$. If $F^{-1}(A)$ is not closed, then there is a compact set $K \subset X$ such that $K \cap\left(X \backslash F^{-1}(A)\right)$ is not open in $K$. Now $F(K)$ is compact and thus $A \cap F(K)$ is compact. Hence, $F^{-1}(A \cap$ $F(K))=C$ is closed. Next we show that $F^{-1}(A) \cap K=C \cap K$. For this

$$
\begin{aligned}
C \cap K & =F^{-1}(A \cap F(K)) \cap K=F^{-1}(A) \cap F^{-1}(F(K)) \cap K \\
& =F^{-1}(A) \cap K .
\end{aligned}
$$

Thus $F^{-1}(A) \cap K$ is relatively closed and so $\left(X \backslash F^{-1}(A)\right) \cap K$ is open in $K$. Thus $F^{-1}(A)$ is closed.

Next we prove another result for $k_{3}$-spaces which is a variation on one of Fuller's results.

THEOREM 4.2. Let $F: X \rightarrow Y$ be a multifunction on a Hausdorff $k_{3}$-space $X$ into a Hausdorff space $Y$. If $F$ is compact preserving and if $F^{-1}(y)$ is closed for all $y \in Y$, then $F$ is upper semicontinuous.

Proof. Let $A \subset Y$ be closed, then in order to show that $F^{-1}(A)$ is closed it is sufficient to show that $F^{-1}(A) \cap K$ is closed for each compact set $K \subset X$. For this note that $F^{-1}(A) \cap K=(F \mid K)^{-1}(A)$, and thus it will suffice to show that $F \mid K$ is u.s.c. Now since $K$ is compact $T_{2}$, it is regular and since $F \mid K$ is closed $(F \mid K$ is compact 
preserving and $Y$ is $T_{2}$ ), $F$ is locally closed with $F^{-1}(y)$ closed for each $y$. Then $F \mid K$ has a closed graph by Theorem 3.4 and thus Theorem 4.1 shows that $F \mid K$ is u.s.c. Hence, $F^{-1}(A) \cap K$ is closed and the theorem follows.

\section{REFERENCES}

1. R. V. Fuller, Relations among continuous and various noncontinuous functions, Pacific J. Math., 25 (1968), 495-509.

2. E. Halfar, Compact mappings, Proc. Amer. Math. Soc., 8 (1957), 828-830.

3. J. L. Kelley, General Topology, D. van Nostrand Co., Princeton, 1955.

4. T. B. Muenzenberger and R. E. Smithson, Continuity and closed functions, to appear.

5. R. E. Smithson, On criteria for continuity, Nieuw archief voor Wiskunde, 14 (1966), 89-92.

Received April, 11975 and in revised form July 15, 1975.

UNIVERSITY OF WYOMING 


\section{PACIFIC JOURNAL OF MATHEMATICS}

\section{EDITORS}

RICHARD ARENS (Managing Editor) University of California

Los Angeles, California 90024

\section{R. A. BEAUMONT}

University of Washington Seattle, Washington 98105
J. DugundjI Department of Mathematics University of Southern Californı Los Angeles, California 90007

D. Gilbarg AND J. Milgram Stanford University Stanford, California 94305

\section{ASSOCIATE EDITORS}

E. F. BECKENBACH

B. H. NeUMANN

F. WOLF

K. YosHidA

\section{SUPPORTING INSTITUTIONS}

UNIVERSITY OF BRITISH COLUMBIA CALIFORNIA INSTITUTE OF TECHNOLOGY UNIVERSITY OF CALIFORNIA MONTANA STATE UNIVERSITY UNIVERSITY OF NEVADA NEW MEXICO STATE UNIVERSITY OREGON STATE UNIVERSITY UNIVERSITY OF OREGON OSAKA UNIVERSITY

\author{
UNIVERSITY OF SOUTHERN CALIFORNIA \\ STANFORD UNIVERSITY \\ UNIVERSITY OF TOKYO \\ UNIVERSITY OF UTAH \\ WASHINGTON STATE UNIVERSITY \\ UNIVERSITY OF WASHINGTON \\ AMERICAN MATHEMATICAL SOCIETY \\ NAVAL WEAPONS CENTER
}




\section{Pacific Journal of Mathematics}

\section{Vol. 61, No. $1 \quad$ November, 1975}

Jiří Adámek, V. Koubek and Věra Trnková, Sums of Boolean spaces represent every

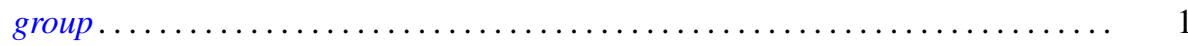

Richard Neal Ball, Full convex l-subgroups and the existence of $a^{*}$-closures of

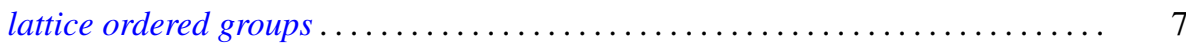

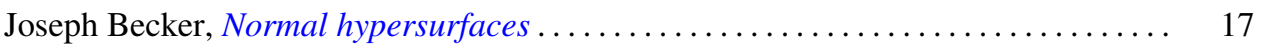

Gerald A. Beer, Starshaped sets and the Hausdorff metric . . . . . . . . . . . . . 21

Dennis Dale Berkey and Alan Cecil Lazer, Linear differential systems with

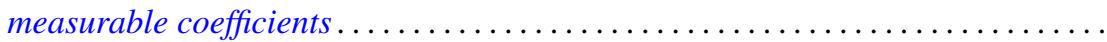

Harald Boehme, Glättungen von Abbildungen 3-dimensionaler

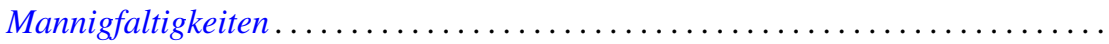

Stephen LaVern Campbell, Linear operators for which $T^{*} T$ and $T+T^{*}$

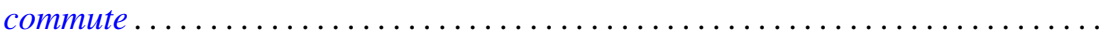

H. P. Dikshit and Arun Kumar, Absolute summability of Fourier series with

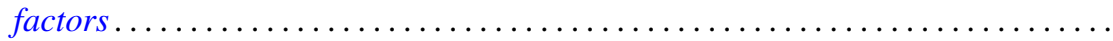

Andrew George Earnest and John Sollion Hsia, Spinor norms of local integral

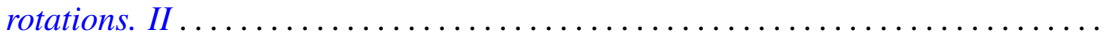

Erik Maurice Ellentuck, Semigroups, Horn sentences and isolic structures .........

Ingrid Fotino, Generalized convolution ring of arithmetic functions . . . . . . . . . . .

Michael Randy Gabel, Lower bounds on the stable range of polynomial rings .......

Fergus John Gaines, Kato-Taussky-Wielandt commutator relations and

characteristic curves

Theodore William Gamelin, The polynomial hulls of certain subsets of $C^{2}$

R. J. Gazik and Darrell Conley Kent, Coarse uniform convergence spaces. . .

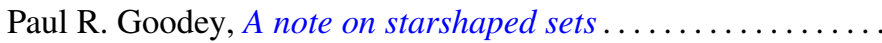

Eloise A. Hamann, On power-invariance

M. Jayachandran and M. Rajagopalan, Scattered compactification for $N \cup\{P\}$. . .

V. Karunakaran, Certain classes of regular univalent functions .

John Cronan Kieffer, A ratio limit theorem for a strongly subadditive set function in a locally compact amenable group .................

Siu Kwong Lo and Harald G. Niederreiter, Banach-Buck measure, density, and uniform distribution in rings of algebraic integers ........

Harold W. Martin, Contractibility of topological spaces onto metric spaces ....

Harold W. Martin, Local connectedness in developable spaces .

A. Meir and John W. Moon, Relations between packing and covering numbers of a tree.

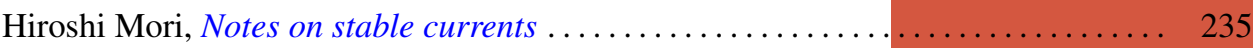

Donald J. Newman and I. J. Schoenberg, Splines and the logarithmic function . . . . 241

M. Ann Piech, Locality of the number of particles operator....

Fred Richman, The constructive theory of $K T$-modules .......

Gerard Sierksma, Carathéodory and Helly-numbers of

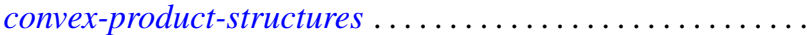

Raymond Earl Smithson, Subcontinuity for multifunctions .... . . 